\title{
On the Estimation of the Leaf Angle Distribution from Drone Based Photogrammetry
}

\section{Xu, Shan}

IEEE

2020

Xu , S , Zaidan , M A , Honkavaara , E, Hakala , T , Viljanen , N , Porcar-Castell , A , Liu , Z

\& Atherton , J 2020 , On the Estimation of the Leaf Angle Distribution from Drone Based Photogrammetry . in IGARSS 2020 - 2020 IEEE International Geoscience and Remote Sensing Symposium . , 9323498 , IEEE International Symposium on Geoscience and Remote Sensing IGARSS, IEEE , pp. 4379-4382, IEEE International Geoscience and Remote Sensing Symposium (IGARSS) , 26/09/2020 . https://doi.org/10.1109/IGARSS39084.2020.9323498

http://hdl.handle.net/10138/337160

https://doi.org/10.1109/IGARSS39084.2020.9323498

acceptedVersion

Downloaded from Helda, University of Helsinki institutional repository.

This is an electronic reprint of the original article.

This reprint may differ from the original in pagination and typographic detail.

Please cite the original version. 


\title{
ON THE ESTIMATION OF THE LEAF ANGLE DISTRIBUTION FROM DRONE BASED PHOTOGRAMMETRY
}

\author{
Shan Xu ${ }^{a, b}$, Martha A.Zaidan ${ }^{c}$, Eija Honkavaara ${ }^{d}$, Teemu Hakala ${ }^{d}$, Niko Viljanen ${ }^{d}$, Albert Porcar- \\ Castell $^{a}$, Zhigang Liu ${ }^{b}$, Jon Atherton ${ }^{a}$ \\ ${ }^{a}$ Optics of Photosynthesis Laboratory, Institute for Atmospheric and Earth System Research/Forest \\ Sciences, Faculty of Agriculture and Forestry, University of Helsinki, 00014 Helsinki, Finland; \\ ${ }^{\mathrm{b}}$ State Key Laboratory of Remote Sensing Science, Jointly Sponsored by Beijing Normal University and \\ Institute of Remote Sensing and Digital Earth of Chinese Academy of Sciences, Beijing 100875, China; \\ ${ }^{\mathrm{c}}$ Institute for Atmospheric and Earth System Research/Physics, Helsinki University, 00560 Helsinki, \\ Finland; \\ ${ }^{\mathrm{d}}$ Department of Remote Sensing and Photogrammetry, Finnish Geospatial Research Institute, National \\ Land Survey of Finland, Geodeetinrinne 2, 02431 Masala, Finland.
}

\begin{abstract}
Leaf angle distribution (LAD) is a key canopy structural parameter, playing an important role in light transfer. LAD can be estimated from fixed point of view photography, however this is time consuming and spatially limited. Recently, Terrestrial LiDAR Scanning (TLS) has been used to estimate LAD through 3D canopy space. The downside of TLS it is more costly than the cameras used in the photographic method. We propose a cost effective method to estimate LAD from drone based photogrammetry. We compare LAD estimates in different water treatment plots. Results show that LAD can be obtained from photogrammetric point clouds. Leaf angles were enhanced in stressed plots, presumably due to wilting. Further, the leaf azimuth distribution was not random but concentrated around 0 and 180 degrees. In summary, drone based photogrammetry can be used to estimate remote sensing parameters such as LAD paving the way for cost effective trait estimation.
\end{abstract}

Index Terms - Leaf angle distribution (LAD), Point clouds, Drone, Photogrammetry

\section{INTRODUCTION}

To increase food production in response to global challenges such as rising population and climate change requires the adoption of modern and sustainable vegetation management practices. The ability to rapidly estimate and accurately monitor the dynamic health status (e.g. photosynthetic rate, stomatal conductance rate, predicted crop yield) of crops at scale is therefore a topic of environmental and economic significance. Remote sensing techniques based on optical or thermal imagery have traditionally filled this niche [1].

The physically-based theory of light transport is integral to the successful application of remote sensing data to the estimation of plant health. This is because architecturalstructural traits such as the vertically integrated density of leaf material, better known as the leaf area index (LAI) determine the radiative transfer (RT) processes within a canopy, influencing the resulting observations [2]. In addition to the LAI, the angular distribution of leaves, or Leaf Angle Distribution (LAD), also plays a significant role in light transfer. In RT theory, the LAD determines the G-function, which corresponds to the fraction of foliage projected on the plane normal to the view zenith direction. The attenuation of light through (a theoretically unclumped) canopy can then be approximated as a function of the product of the LAD-derived $\mathrm{G}$ function and the LAI [3]. This is the gap fraction $(P)$ theory that is used to estimate LAI from field observations [3, 4]:

$P(\theta)=\exp \left(\frac{-G(\theta) * L A I}{\cos (\theta)}\right)$

where, $\theta$ is the view zenith angle. From a functional point of view the LAD is important as a canopy trait that contains information on the water status of the plant (i.e. the degree of wilting), circadian rhythms and sun tracking or avoidance strategies $[5,6,7]$.

Despite its significant importance, LAD is difficult to measure. For this reason, LAD is often assumed to be spherical (isotropic $\mathrm{G}$ function), or assigned an alternative archetypal distribution in order to simplify the process of RT modelling [8]. It is possible to measure zenith angles using a technique based on leveled digital photography (LDP) [9]. In addition to ground based studies, the LDP method has also been applied using a drone platform [10], but it is time consuming and requires manual visual inspection prior to processing [4]. The LDP method also ignores the 3D component of the angular distribution i.e. the azimuthal angle.

Terrestrial LiDAR scanning (TLS) is increasingly used to measure canopy structure using point cloud data. Such systems capture detailed 3-D structural information of the 
canopy. Recently, Vicari et al. [11] proposed a fast and simple method for the estimation of the LAD from TLS point clouds based on leaf plane fitting using a Singular Value Decomposition approach. The drawback of TLS systems is cost, which can be prohibitive for small budget field campaigns. Photogrammetric point clouds provide a lowcost alternative to TLS systems, requiring only consumer cameras and image processing software [12]. On the ground, photogrammetry has been used to estimate LAD demonstrating the viability of the technique [13].

Again, primarily due to low cost and the low technical barrier to entry, drones are increasingly used as remote sensing platforms to supplement and extend (in terms of spatial resolution) the capabilities of crewed aircraft and satellite remote sensing systems [14]. Drone based photogrammetric imaging techniques utilizing dense image matching is a promising tool to collect canopy structure information with 3D information. Previous studies have focused on using drone based photogrammetry to estimate canopy height [15]. In this study, we aim to use drone based photogrammetry to estimate LAD in a water stress experiment.

\section{METHODS}

Remote sensing datasets were collected on $11^{\text {th }}, 17^{\text {th }}$ and $25^{\text {th }}$ of July in 2018, using the Finnish Geospatial Research Institute's drone that had Gryphon Dynamics quadcopter frame with Pixhawk autopilot. The drone was equipped with Applanix APX-15-EI UAV positioning system, that consist of multiband GNSS and Inertial Measurement Unit (IMU), and two Sony A7R II digital cameras with a Sony FE 35mm f/2.8 ZA Carl Zeiss Sonnar T* lens. Cameras were mounted $+15^{\circ}$ and $-15^{\circ}$ angles in stabilized rack with APX-15 EI UAV that recorded camera location and angles for each image. In addition to the stablized rack, image quality was further increased by flying at low speeds (approximately $2.5 \mathrm{~m} / \mathrm{s}$ ).

Photogrammetric data (Fig. 1) were produced using dronebased imagery collected using the protocol described in Nevalainen et al. [12]. We used flying height of $50 \mathrm{~m}$ resulting of Ground Sampling Distance (GSD) of $0.64 \mathrm{~cm}$, respectively. We carried out double grid flight path that consisted of 6 north-south flight lines and 9 east-west flight lines. Thus, the area of interest was captured in more than nine overlapping oblique images. We used observation data from National Land Survey of Finland (NLS) RINEX service to calculate Post Processed Kinematic (PPK) GNSS solutions and angles for each camera in Applanix POSPac UAV (Applanix, Richmond Hill, Ontario, Canada) software.

Photogrammetric data were processed using Agisoft PhotoScan Professional commercial software (AgiSoft LLC, St. Petersburg, Russia), following similar workflow as described in Viljanen et al [15]. In image orientation process high quality setting with 40,000 key points and 4,000 tie points limit per image was selected. Dense point cloud calculation was done using ultrahigh quality and mild depth filtering, resulting of point densities of $2.26-2.43$ points $/ \mathrm{cm}$ and re-projection errors of $0.9-1.14$, respectively.

LAD was retrieved for 4 different water treatment plots. Fig. 1 shows an example of a dense point cloud from one of these plots. From here, we use P1(+), P2(-), P3(-), P4(+) to represent the 4 plots, where sign + means water controlled, sign - means water stressed. Plots $\mathrm{P} 1$ and $\mathrm{P} 2$ are from $17^{\text {th }}$ July and P3 and P4 from the $25^{\text {th }}$ July.

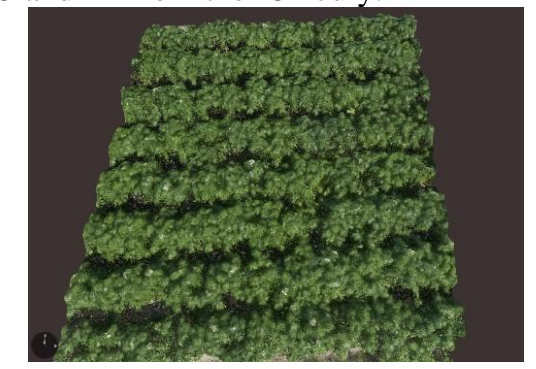

Fig. 1 Example of dense point clouds data

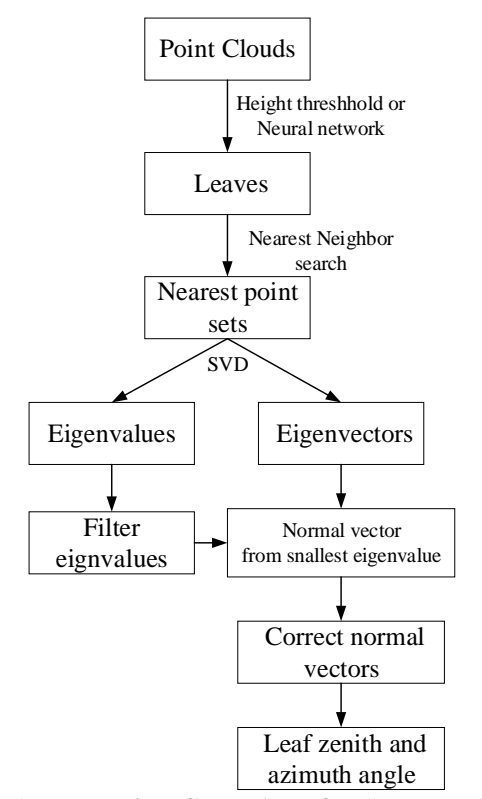

Fig. 2 Processing flow chart for SVD method

In addition to dense points clouds, Photoscan can also output normal data, which we used to estimate LAD directly using equations 2 and 3 . We also chose to re-calculate normals from the dense point clouds using SVD method [11]. The SVD method has a potential smoothing effect, and is additionally useful in cases (e.g. archived datasets) where normals have not been saved. The two methods are referred to as Photoscan normals and SVD normals in the sections below.

The LAD estimation algorithm starts by assuming leaf angle can be obtained by accumulating all valid planes fitted to points in a leaf point cloud, as in based on Vicari's TLS method [11]. Algorithm flow chart is shown in Fig. 2. To exclude soil, we first needed to classify leaves and soil. Here, we tested two methods: first setting a plot-dependent height threshold, and second using an artificial neural network 
algorithm. The results shown here relate to the height thresholding as we continue to test and develop the neural network approach.

After classification, a Nearest Neighbors search (kNN) algorithm was used to approximate the leaf plane around each point in the leaf cloud. The kNN parameter (number of neighbors) should be a compromise between a neighbor-hood of points small enough and the number of points sufficient to minimize the impact of noise. To determine optimal kNN value, a sensitivity analysis was performed for different value from 5 to 25 points. Here, a value of 15 points was selected.

Singular Value Decomposition (SVD) was used to perform plane fitting on each subset of points and filter outliers by setting ratio between third eigenvalue and the sum of all three eigenvalues as 0.15 . Finally, plane normal vectors of each plane, leaf inclination angle $(\theta)$ and leaf azimuth angle $(\phi)$ were produced by calculating the angle between normal vectors $(n)$ and a zenith vector defined $(z)$ as $(0,0,1)$ : $\theta=\arccos \frac{n \cdot z}{|n||z|}$

$\phi=\arccos \frac{n^{p} \cdot \mathrm{z}^{p}}{\left|n^{p}\right|\left|z^{p}\right|}$

where $n^{p}$ is the project vectors of normal vectors $(n)$ on the $(\mathrm{x}, \mathrm{y})$ plane, and $z^{p}$ is vector $(0,1)$.

Vicari's algorithm coded in Python can be found from https://github.com/mattbv/lidar_leaf_properties [11].

Post estimation, we fitted the two parameter Beta distribution function to LAD zenith angles using Matlab numerical optimization fmincon method.

To validate retrieved LAD estimation accuracy, we measured average leaf angle (ALA) using LDP measurements for 4 plots. For LDP measurements, we choose 12-15 leaves to estimate ALA from photographs. RMSE were calculated between the retrieved and the measured ALA.
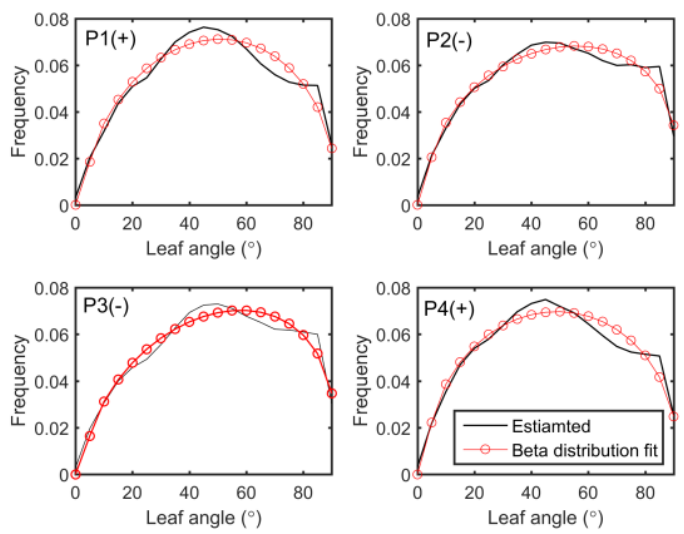

Fig. 3 Retrieved LAD and fitted Beta distribution of 4 plots using SVD method. Note that SVD derived distributions are artificially limited to $90^{\circ}$, which results in a bias in ALA and a visible kink in the distributions close to $80^{\circ}$.

\section{RESULTS AND CONCLUSIONS}

Results of leaf angle distributions retrieved from point cloud data are shown in Fig. 3 (zeniths) and Fig.4 (azimuths and zeniths). Different water treatment plots present differing LADs. There are smaller leaf zenith angles for controlled relative to stressed plots, which signifies a greater degree of wilting (Fig. 3). Result also show that the leaf azimuth angle distribution isn't random, but concentrated around 0 and 180 degree. Hence, for our row crops, the assumption of randomly distributed azimuth angle (e.g. spherical archetype) does not hold. In addition, this assumption will also impact $\mathrm{G}$ function estimation, hence LAI estimation based on physical theory.
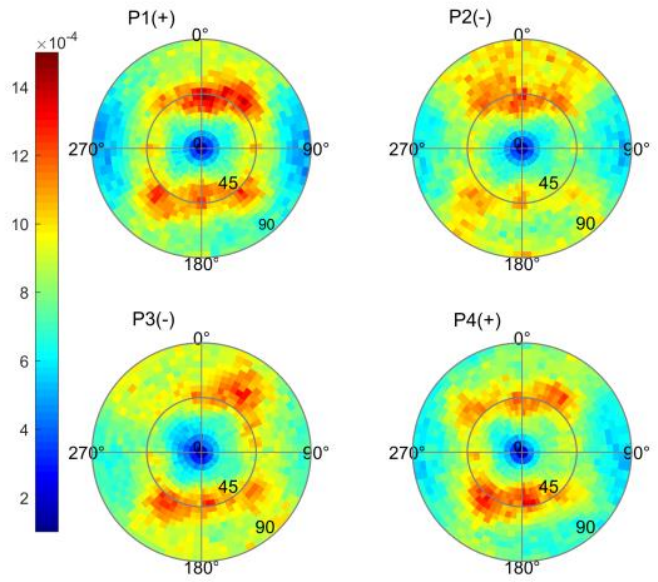

Fig. 4 Leaf zenith angle and azimuth angle distribution in polar coordinates of 4 plots from SVD method.

Although we only have four points, the RMSE of average leaf angle for SVD method is about 2.3 degree and 7.0 degrees for Photoscan as shown in Fig. 5. Neither relationship fell on the 1:1 line. Clearly, the canopy penetration offered by (visible) photogrammetric methods is limited to outside layers and/or unoccluded objects. Systematic errors could also occur because we compared LDP collected at a slightly different time and fixed height to vertically integrated LAD collected from the drone. Random errors could also influence the result, hence more validation data is required to assess the method in the future with greater consideration to the LDP sampling procedure, especially if the method is to be applied to structurally complex woody or occluded canopies such as mature trees. Importantly, we also found a low bias in the SVD ALA estimate. This was caused by the SVD method capping the zenith angle distribution to angles below $90^{\circ}$ (Fig. 3 ) so that only upwards away from ground pointing vectors result from the SVD code. This has implications if the target contains many near-vertical planes e.g. drooping leaves. We aim to improve the SVD algorithm to account for this using directional information from the Photoscan vectors. This would combine the smoothing benefit of the SVD with correct orientation of the vectors from the Photoscan method. Until this issue is solved, we strongly recommend using the normal vectors produced by Photoscan if available rather than the SVD method, especially if there are many vertical elements in the scene. Finally, the higher RMSE in Photoscan relative to SVD method could partially be explained by the 
fact that our validation data were also possibly biased low as we only measured a few samples. As stated above, more spatially comprehensive validation data is required in future studies. Further work should also examine the retrieval and combination of LAD, clumping indices and LAI from photogrammetry.

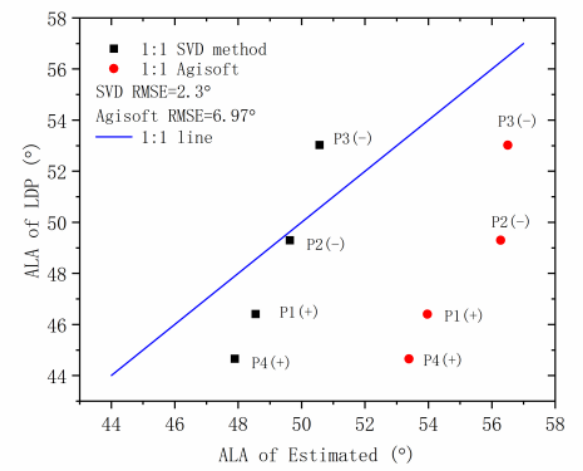

Fig. 5 Average leaf angle of LDP Vs estimated LAD from photogrammetry.

\section{ACKNOLEDGEMENTS}

This work was supported by the Academy of Finland (grant number 288039, 304097). Shan Xu was also supported by the China Scholarship Council (CSC) under grant 201806040163 and by the Natural Science Foundation of China (grant numbers 41571409, 41541043) during this work.

\section{REFERENCES}

[1] P. J. Zarco-Tejada, V. González-Dugo, \& J. A. Berni, "Fluorescence, temperature and narrow-band indices acquired from a UAV platform for water stress detection using a micro-hyperspectral imager and a thermal camera", Remote sensing of environment, 117, pp. 322-337, 2012.

[2] J. Verrelst, J. P. Rivera, C. van der Tol, F. Magnani, G. Mohammed, and J. Moreno, "Global sensitivity analysis of the SCOPE model: What drives simulated canopy-leaving sun-induced fluorescence?", Remote Sensing of Environment, 166, pp. 8-21, 2015.

[3] N. J. Breda, "Ground-based measurements of leaf area index: a review of methods, instruments and current controversies", Journal of experimental botany, 54(392), pp. 2403-2417, 2003.

[4] J. Pisek, O. Sonnentag, A. D. Richardson, and M. Mõttus, "Is the spherical leaf inclination angle distribution a valid assumption for temperate and boreal broadleaf tree species?", Agricultural and Forest Meteorology, 169, pp. 186-194, 2013.

(C) 2021 IEEE. Personal use of this material is permitted. Permission from IEEE must be obtained for all other uses, in any current or future media, including reprinting/republishing this material for advertising or promotional purposes, creating new collective works, for resale or redistribution to servers or lists, or reuse of any copyrighted component of this work in other works.
[5] G. Puglielli, L. Gratani, \& L. Varone, "Leaf rolling as indicator of water stress in cistus incanus from different provenances", BioRxiv, 131508, 2017.

[6] M. J. Yanovsky, M. Izaguirre, J. A. Wagmaister, C. Gatz, S. D. Jackson, B. Thomas, \& J. J. Casal, "Phytochrome A resets the circadian clock and delays tuber formation under long days in potato", The Plant Journal, 23(2), pp. 223-232, 2000 .

[7] C. E. Lovelock and B. F. Clough, "Influence of solar radiation and leaf angle on leaf xanthophyl concentrations in mangroves”, Oecologia, 91(4), pp. 518-525, 1992.

[8] C. Tol, W. Verhoef, J. Timmermans, A. Verhoef, and Z. $\mathrm{Su}$, "An integrated model of soil-canopy spectral radiances, photosynthesis, fluorescence, temperature and energy balance”, Biogeosciences, 6(12), pp. 3109-3129, 2009.

[9] Y. Ryu, O. Sonnentag, T. Nilson, R. Vargas, H., Wenk, R. Kobayashi, and D. D. Baldocchi, "How to quantify tree leaf area index in an open savanna ecosystem: a multi-instrument and multi-model approach", Agricultural and Forest Meteorology, 150(1), pp. 63-76, 2010.

[10] B. E. McNeil, J. Pisek, H. Lepisk, and E. A. Flamenco, "Measuring leaf angle distribution in broadleaf canopies using UAVs", Agricultural and Forest Meteorology, 218, pp. 204-208, 2016.

[11] M. B. Vicari, J. Pisek, and M. Disney, "New estimates of leaf angle distribution from terrestrial LiDAR: Comparison with measured and modelled estimates from nine broadleaf tree species", Agricultural and forest meteorology, 264, pp. 322-333, 2019.

[12] O. Nevalainen, E. Honkavaara, S. Tuominen, N. Viljanen, T. Hakala, X. Yu, ... and A. Tommaselli, "Individual tree detection and classification with UAV-based photogrammetric point clouds and hyperspectral imaging", Remote Sensing, 9(3), 185, 2017.

[13] H. Wang, W. Zhang, G. Zhou, G. Yan and N. Clinton, "Image-based 3D corn reconstruction for retrieval of geometrical structural parameters", International Journal of Remote Sensing, 30(20), pp. 5505-5513, 2009.

[14] Nex, F. and Remondino, F., "UAV for 3D mapping applications: a review", Applied geomatics, 6(1), pp.1-15, 2014.

[15] N. Viljanen, E. Honkavaara, R. Näsi, T. Hakala, O. Niemeläinen, and J. Kaivosoja, "A novel machine learning method for estimating biomass of grass swards using a photogrammetric canopy height model images and vegetation indices captured by a drone", Agriculture, 8(5), pp. 70, 2018. 